\title{
Validity of gait parameters for hip flexor contracture in patients with cerebral palsy
}

\author{
Sun Jong Choi ${ }^{1}$, Chin Youb Chung ${ }^{2 *}$, Kyoung Min Lee ${ }^{2}$, Dae Gyu Kwon², Sang Hyeong Lee ${ }^{3}$, Moon Soek Park ${ }^{2}$
}

\begin{abstract}
Background: Psoas contracture is known to cause abnormal hip motion in patients with cerebral palsy. The authors investigated the clinical relevance of hip kinematic and kinetic parameters, and 3D modeled psoas length in terms of discriminant validty, convergent validity, and responsiveness.

Methods: Twenty-four patients with cerebral palsy (mean age 6.9 years) and 28 normal children (mean age 7.6 years) were included. Kinematic and kinetic data were obtained by three dimensional gait analysis, and psoas lengths were determined using a musculoskeletal modeling technique. Validity of the hip parameters were evaluated.

Results: In discriminant validity, maximum psoas length (effect size $r=0.740$ ), maximum pelvic tilt $(0.710$ ), maximum hip flexion in late swing (0.728), maximum hip extension in stance (0.743), and hip flexor index (0.792) showed favorable discriminant ability between the normal controls and the patients. In convergent validity, maximum psoas length was not significantly correlated with maximum hip extension in stance in control group whereas it was correlated with maximum hip extension in stance $(r=-0.933, p<0.001)$ in the patients group. In responsiveness, maximum pelvic tilt ( $p=0.008$ ), maximum hip extension in stance $(p=0.001$ ), maximum psoas length $(p<0.001)$, and hip flexor index $(p<0.001)$ showed significant improvement post-operatively.
\end{abstract}

Conclusions: Maximum pelvic tilt, maximum psoas length, hip flexor index, and maximum hip extension in stance were found to be clinically relevant parameters in evaluating hip flexor contracture.

\section{Background}

Hip flexion deformity or spasticity is a cause of the abnormal gait observed in cerebral palsy patients. Hip flexor spasticity was reported to cause dynamic restriction of hip extension in the terminal stance and become fixed hip flexion contracture with age in those patients [1-3]. The psoas muscle is a primary cause of hip flexion contracture $[4,5]$ and has been known to be associated with increased anterior pelvic tilt, crouch gait, hip instability and lumbar lordosis, which can eventually cause spondylosis and back pain [1,4,6-8]. The psoas muscle plays an important role in advancing the lower leg during normal gait [4], whereas the dysphasic activity of the hip flexor muscle opposes and limits hip

\footnotetext{
* Correspondence: chungcy55@gmail.com

${ }^{2}$ Department of Orthopedic Surgery, Seoul National University Bundang Hospital, 300 Gumi-Dong, Bundang-Gu,Sungnam, Kyungki 463-707, Republic of Korea

Full list of author information is available at the end of the article
}

extension in patients with cerebral palsy [4,9-11], which reduces the stride length and gait efficacy.

Despite the role of this muscle in the pathologic gait, the surgical indications of psoas lengthening are somewhat vague. Furthermore, although several kinematic and kinetic variables were shown to represent hip motion during gait and those variables were used to report changes after single event multilevel surgery in patients with cerebral palsy, the clinical relevance of those variables measuring the hip flexor function is unclear.

After 3D modeled muscle length calculated from kinematic data of gait analysis was devised, it was believed that this could be especially useful in measuring dynamic length of multijoint muscle during gait because reflecting the multijoint movement is not easy to follow [12]. Several studies have investigated 3D modeled psoas length [13-15], but its clinical relevance has not been sufficiently verified.

The kinematic and kinetic data of hip motion as well as the 3D psoas length need to be evaluated accurately

\section{() Biomed Central}

(c) 2011 Choi et al; licensee BioMed Central Ltd. This is an Open Access article distributed under the terms of the Creative Commons Attribution License (http://creativecommons.org/licenses/by/2.0), which permits unrestricted use, distribution, and reproduction in any medium, provided the original work is properly cited. 
for clinical use. This study examined the validity of kinematic and kinetic variables measuring the hip flexor function and the 3D modeled psoas length by 1 ) discriminating the pathologic gait from the normal gait (discriminant validity), 2) correlating those variables (convergent validity), and 3) analyzing post-operative changes (responsiveness).

\section{Methods}

\section{Inclusion/Exclusion Criteria}

This retrospective study was performed at a tertiary referral center for cerebral palsy and was approved by the institutional review board. The study was designed to include a group of normal children and a group of patients with cerebral palsy. For the group of normal children, volunteers aged from 5 to 15 years old were recruited. The exclusion criteria were known neuromuscular disease and an abnormality of lower limb alignment. For the study group, patient selection was based on the medical records since 1997. In order to have a homogenous group of the patients with cerebral palsy, the following inclusion criteria were used: 1) ambulatory patients with spastic diplegia (GMFCS level I-II, gross motor function classification system [16], who had the representative gait pattern consisting of a jump gait pattern [17] with intoeing, equinus, stiff knee, and femoral antetorsion, which is one of the most representative gait patterns of diplega; 2) patients who underwent bilateral single event multilevel surgery (bilateral tendo-Achilles lengthening, distal hamstring lengthening, rectus femoris transfer, femoral derotational osteotomy); 3) a follow-up period of more than one year; 4) the pre-operative and post-operative gait analysis; and 5) 5-15 years of age. The exclusion criteria were patients with a history of gait corrective surgery or selective dorsal rhizotomy, neuromuscular diseases other than cerebral palsy, an asymmetrical gait pattern and surgical procedures other than the index procedures. The demographic data, physical examination (including Thomas test [18]), and gait parameters of the patients, including gender, age, GMFCS level, cadence, step length, and walking speed, were collected. Informed consent for the retrospective review of the gait analysis data of patients and control group was waived by the institutional review board at our hospital.

\section{Kinematic and kinetic data}

The gait analysis laboratory was equipped with a Vicon 370 (Oxford Metrix, Oxford, UK) system consisting of seven CCD cameras and two force plates. Motion was captured while the subjects walked barefoot on a ninemeter walkway, and the kinematic and kinetic data were obtained, which were averaged by three trials. The hip flexion and extension, hip rotation, and pelvic tilt were the key kinematic variables. The kinetic data including time of crossover in the hip flexion-extension moment and the power burst of hip flexor in the late stance were obtained. The hip flexor index was calculated from the kinematic and kinetic data of the hip and pelvic motion, which were maximum pelvic tilt, pelvic tilt range, maximum hip extension in stance, and late stance power burst of hip joint (H3) [19].

\section{D modeled psoas length}

The psoas length was obtained using interactive musculoskeletal modeling [20] software (SIMM, Motion Analysis Corporation, Santa Rosa, CA) (Figures 1 and 2). The psoas length was determined to be between the muscular origin and insertion, which were the transverse process of the lumbar spine and lesser trochanter of the femur, respectively. However, in this study, spine motion was not included. Calculated average psoas origin was used, and calculated average pelvic brim was used as via

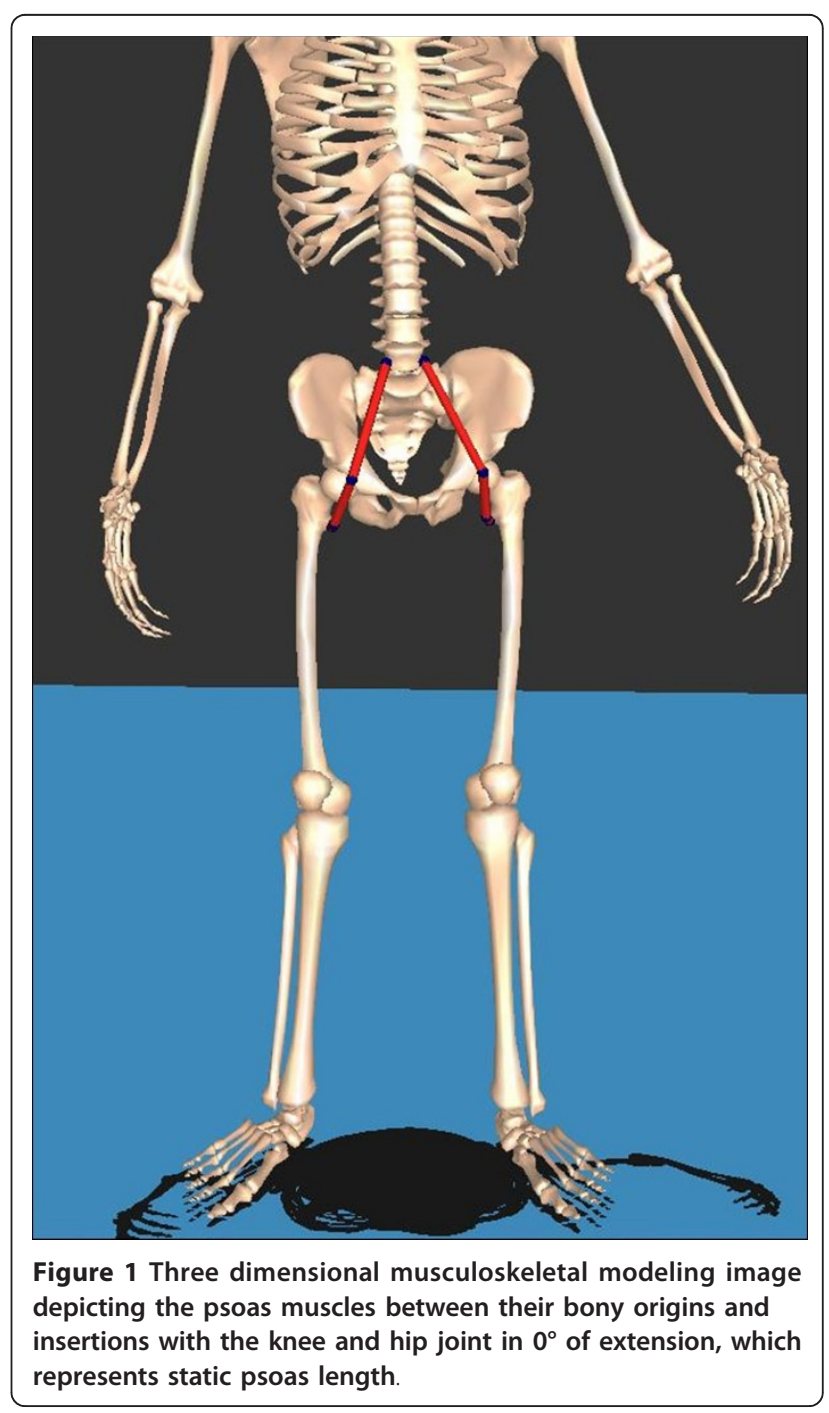




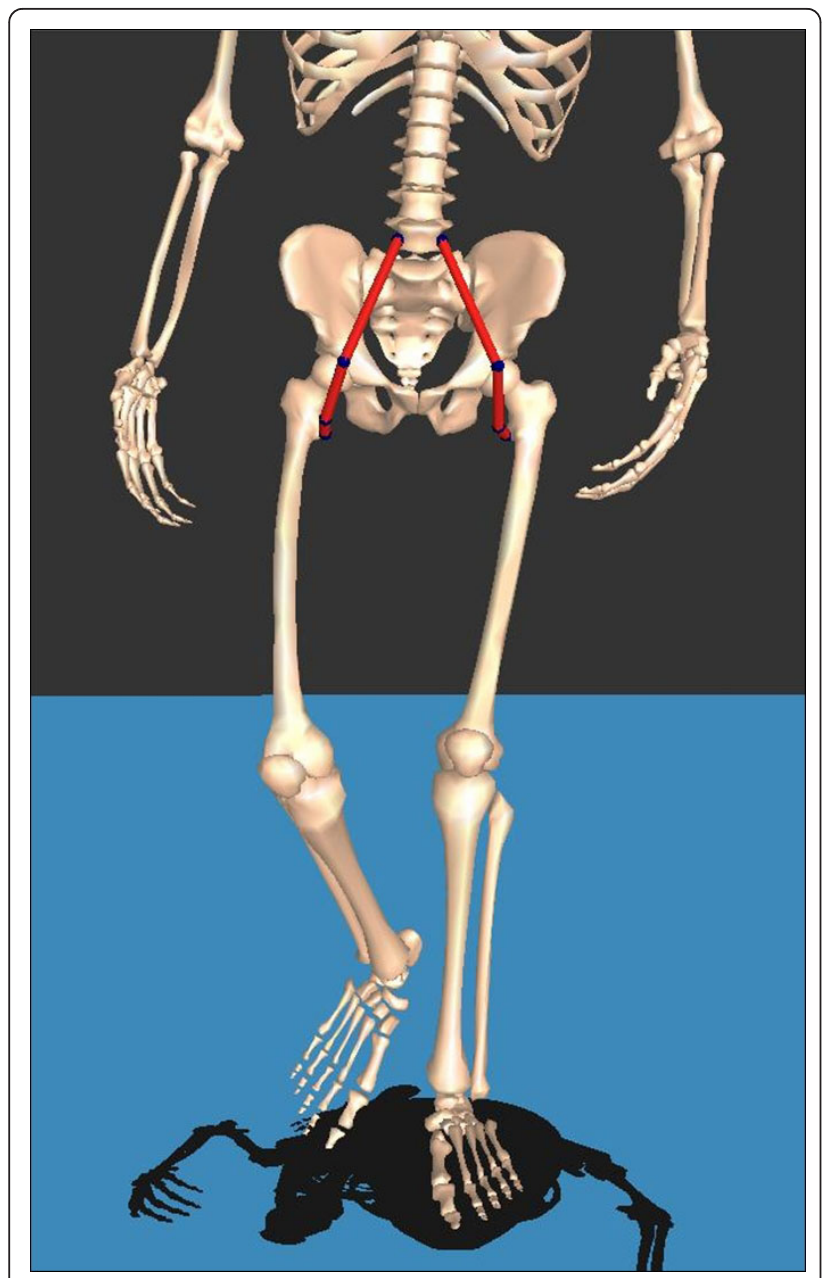

Figure 2 Psoas length (distance between its bony origin and insertion) changed throughout the gait cycle, which is dynamic psoas length.

points. The anatomic points were calculated from the kinematic data of femur and pelvis. Although psoas is a multijoint muscle, only hip angles were reflected in its length. The psoas length was standardized by dividing the calculated psoas length during gait by the muscle length when the subjects were in a simulated anatomic position. This standardized psoas length was recorded continuously during the gait cycle (Figure 3) and included for analysis.

\section{Validity of kinematic and kinetic variables, and psoas length in hip flexor function}

There are no gold standards for measuring hip flexor function during gait. Therefore, the validity of kinematic and kinetic data regarding hip flexor function relies on the content validity and construct validity. Construct validity is comprised of the discriminant validity and convergent validity. The discriminant validity [21] is one

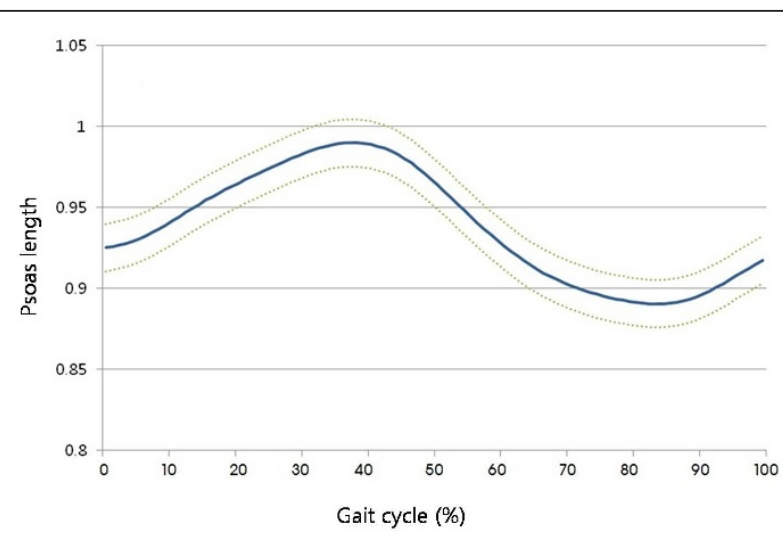

Figure 3 Standardized psoas length was calculated and depicted throughout the gait cycle, which is dynamic psoas length divided by static psoas length

facet of the construct validity, and reflects the degree to which an instrument can distinguish between or among different concepts or constructs [22]. This is the ability to detect clinically relevant difference. In this study, effect-size $r$ [23] between the normal control and the patient groups were assessed as in previous studies [24-27]. Convergent validity [21,28] which is another type of construct validity, occurs when the scales of a measurement correlate as expected with the related scales of another measurement. In this study, the 3D modeled psoas lengths were compared with the kinematic and kinetic hip parameters representing hip and pelvic motion. Responsiveness [29] was tested by comparing the pre-operative and post-operative variables.

\section{Statistical Analysis}

One of the principal variables in this study was the psoas length on which we had few previous studies that we could refer to. We assumed that $1 \%$ of difference in psoas length between the control and patient groups would be clinically relevant, and prior power analysis (alpha error 0.05 , power 0.8 ) revealed that over 17 subjects would be needed on each group. The average of the variables of right and left legs were used for data analysis to ensure data independence.

Statistical analysis was performed using SPSS Ver. 15.0 (SPSS, Chicago, Illinois). The normal distribution of the data was tested using a Kolmogorov-Smirnov test. The discriminant validity was assessed by the effect-size $r$ [23] for the kinematic and kinetic variables and psoas length. The Effect size is a name given to a family of indices that measures the magnitude of a certain effect and is generally measured in two ways: as the standardized difference between two means, or as the correlation between the independent variable classification and individual scores on the dependent variable. 
This correlation is called the effect size correlation (effect-size $r$ ) and was used for the discriminant validity in this study. Correlations between each of the kinematic and kinetic variables and psoas length were analyzed using a Pearson's correlation test for convergent validity. The comparison of the data between the patients and normal controls was performed using a t-test, and the post-operative changes in the patients were analyzed using a paired t-test. A $\mathrm{p}$ value $<0.05$ was considered significant. For multiple testing, statistical significance was adjusted for family wise error.

\section{Results}

Twenty-four patients with cerebral palsy were finally included in this study. The mean age of the patients was 6.9 years (SD 1.6 years), and there were 15 males and 9 females. The GMFCS levels were I in 15 patients and II in 9 patients. The mean age of the 28 normal controls was 7.6 years (SD 2.4 years), and there were 17 males and 11 females. The mean age and gender ratio were not significantly different between the two groups $(\mathrm{p}=$ 0.222 and $\mathrm{p}=0.973)($ Table 1$)$.

\section{Discriminant validity of kinematic and kinetic data, and psoas length}

The discriminant validity between the patients and normal control group was highest in hip flexor index (effect size $r=0.792$ ) followed by maximum hip extension in stance (0.743), maximum psoas length (0.740), maximum hip flexion in late swing (0.728) and maximum pelvic tilt (0.710). Kinetic data, including the time of crossover in hip flexion-extension moment (0.059) and power burst of hip flexor in late stance (0.020), showed an unsatisfactory discriminant validity (Table 2).

\section{Convergent validity of kinematic and kinetic data, and psoas length}

In the normal control group, the correlation coefficient between the maximum psoas length and maximum hip extension in stance was $-0.420(p=0.065)$. The

Table 1 Demographic data and gait parameters

\begin{tabular}{llll}
\hline & Patients & Normal controls & $\boldsymbol{p}$ \\
\hline $\mathrm{N}$ & 24 & 28 & \\
Age (years) & $6.9(1.6)$ & $7.6(2.4)$ & 0.222 \\
Sex (M:F) & $15: 9$ & $17: 11$ & 0.973 \\
Follow up period (years) & $1.1(0.2)$ & - & \\
GMFCS level (I/II) & $15 / 9$ & - & \\
Gait parameters & & & 0.001 \\
$\quad$ Cadence (No./min) & $101.2(14.2)$ & $112.5(12.9)$ & $<0.001$ \\
$\quad$ Step length (cm) & $35.3(6.2)$ & $53.0(9.2)$ & $<0.001$ \\
$\quad$ Walking speed (cm/s) & $59.9(13.5)$ & $99.9(16.9)$ &
\end{tabular}

Data are presented as mean (SD).
Table 2 Discriminant validity of hip parameters

\begin{tabular}{|c|c|c|c|c|}
\hline & $\begin{array}{l}\text { Cerebral } \\
\text { palsy }\end{array}$ & $\begin{array}{l}\text { Normal } \\
\text { controls }\end{array}$ & $p$ & $\begin{array}{l}\text { Effect } \\
\text { size }(r)\end{array}$ \\
\hline Thomas test $\left({ }^{\circ}\right)$ & $7.4(6.8)$ & $0.6(1.9)$ & $<0.001$ & 0.561 \\
\hline \multicolumn{5}{|l|}{ Pelvic tilt $\left(^{\circ}\right)$} \\
\hline maximum & $21.9(4.9)$ & $12.1(4.8)$ & $<0.001$ & 0.710 \\
\hline minimum & $12.6(5.9)$ & $6.9(3.9)$ & 0.008 & 0.497 \\
\hline range & $9.4(3.5)$ & $5.2(2.5)$ & 0.002 & 0.562 \\
\hline mean & $17.5(5.1)$ & $9.5(4.1)$ & $<0.001$ & 0.654 \\
\hline $\begin{array}{l}\text { Max hip extension in } \\
\text { stance }\left({ }^{\circ}\right)\end{array}$ & $-0.5(6.1)$ & $11.1(4.2)$ & $<0.001$ & 0.743 \\
\hline $\begin{array}{l}\text { Max hip flexion in late } \\
\text { swing }\left({ }^{\circ}\right)\end{array}$ & $50.4(5.9)$ & $38.2(5.5)$ & $<0.001$ & 0.728 \\
\hline \multicolumn{5}{|l|}{ Hip rotation $\left({ }^{\circ}\right)$} \\
\hline maximum & $12.8(8.2)$ & $12.8(9.9)$ & 0.997 & 0.005 \\
\hline minimum & $0.2(9.2)$ & $-11.8(13.2)$ & 0.005 & 0.467 \\
\hline range & $12.6(4.1)$ & $24.7(11.5)$ & 0.009 & 0.573 \\
\hline mean & $6.3(8.9)$ & $0.1(10.0)$ & 0.086 & 0.308 \\
\hline \multicolumn{5}{|l|}{ Psoas length (\%) } \\
\hline maximum & $99.2(1.3)$ & $101.5(0.8)$ & $<0.001$ & 0.740 \\
\hline minimum & $87.5(1.4)$ & $90.4(1.7)$ & $<0.001$ & 0.684 \\
\hline range & $11.7(1.5)$ & $11.1(1.2)$ & 0.126 & 0.212 \\
\hline mean & $93.6(1.3)$ & $96.0(1.3)$ & $<0.001$ & 0.676 \\
\hline TOC (\%) & $28.2(11.5)$ & $27.0(8.6)$ & 0.767 & 0.059 \\
\hline H3 (W/kg) & $0.3(0.4)$ & $0.3(0.4)$ & 0.920 & 0.020 \\
\hline $\mathrm{HFI}$ & $5.9(1.4)$ & $1.9(1.7)$ & $<0.001$ & 0.792 \\
\hline
\end{tabular}

TOC, time of cross over in hip flexion/extension moment; $\mathrm{H} 3$, late swing power burst in hip joint flexion/extension power; HFI, hip flexor index.

Data are presented as mean (SD).

maximum psoas length showed correlation coefficients of $0.601,-0.651$, and -0.448 with the step length, time of crossover in hip flexion-extension moment, and hip flexor index, respectively. The minimum psoas length showed no significant correlation with the kinematic and kinetic variables (Table 3).

In the patients group, the maximum psoas length showed a significant correlation with the maximum hip extension in stance $(\mathrm{r}=-0.933, \mathrm{p}<0.001)$. The correlation coefficient between the maximum psoas length and hip flexor index was $-0.467(\mathrm{p}=0.001)$. There was no significant correlation between the maximum psoas length and step length (Table 4). Thomas test did not show significant correlation with maximum psoas length in control and patient groups.

\section{Responsiveness of kinematic and kinetic data, and psoas length}

The maximum pelvic tilt, maximum hip extension in stance, maximum psoas length and hip flexor index showed significant improvement after surgery $(\mathrm{p}=$ $0.008, \mathrm{p}=0.001, \mathrm{p}<0.001$, and $\mathrm{p}<0.001$ respectively). There was no significant post-operative change in the range of psoas lengths $(\mathrm{p}=0.158)$ and power 
Table 3 Correlation coefficients between psoas length and gait parameters in control group

\begin{tabular}{|c|c|c|c|c|}
\hline & Max PL & Min PL & Range PL & Mean PL \\
\hline Thomas test $\left({ }^{\circ}\right)$ & -0.253 & $-0.416^{*}$ & $0.407^{*}$ & $-0.450 *$ \\
\hline \multicolumn{5}{|l|}{ Pelvic tilt $\left({ }^{\circ}\right)$} \\
\hline maximum & $-0.576^{*}$ & -0.206 & 0.050 & -0.399 \\
\hline minimum & $-0.595^{*}$ & -0.141 & -0.018 & -0.296 \\
\hline range & -0.110 & -0.140 & 0.108 & -0.240 \\
\hline mean & $-0.672 *$ & -0.226 & 0.044 & -0.420 \\
\hline $\begin{array}{l}\text { Max hip extension in } \\
\text { stance }\left({ }^{\circ}\right)\end{array}$ & -0.420 & -0.029 & -0.082 & -0.201 \\
\hline $\begin{array}{l}\text { Max hip flexion in late } \\
\text { swing }\left({ }^{\circ}\right)\end{array}$ & -0.312 & -0.327 & 0.238 & -0.302 \\
\hline \multicolumn{5}{|l|}{ Hip rotation $\left(^{\circ}\right)$} \\
\hline maximum & 0.140 & -0.098 & 0.133 & -0.172 \\
\hline minimum & -0.316 & -0.101 & 0.015 & -0.233 \\
\hline range & $0.532^{*}$ & 0.012 & 0.128 & 0.088 \\
\hline mean & -0.128 & -0.091 & 0.055 & -0.226 \\
\hline TOC (\%) & $-0.651^{*}$ & -0.085 & -0.107 & -0.344 \\
\hline H3 (W/kg) & 0.140 & -0.305 & 0.326 & -0.234 \\
\hline $\mathrm{HFI}$ & $-0.448^{*}$ & -0.081 & -0.039 & -0.269 \\
\hline Cadence (No./min) & -0.278 & -0.208 & 0.131 & -0.288 \\
\hline Step length $(\mathbf{c m})$ & $0.601 *$ & 0.206 & -0.044 & 0.355 \\
\hline Walking speed $(\mathrm{cm} / \mathrm{s})$ & $0.511^{*}$ & 0.149 & -0.011 & 0.232 \\
\hline
\end{tabular}

TOC, time of cross over in hip flexion/extension moment; $\mathrm{H3}$, late swing power burst in hip joint flexion/extension power; $\mathrm{HFI}$, hip flexor index; ${ }^{*}, \mathrm{p}<0.05$.

Table 4 Correlation coefficients between psoas length and gait parameters in patients group

\begin{tabular}{|c|c|c|c|c|}
\hline & Max PL & Min PL & Range PL & Mean PL \\
\hline Thomas test $\left(^{\circ}\right)$ & -0.116 & $0.408^{*}$ & $-0.476^{*}$ & 0.200 \\
\hline \multicolumn{5}{|l|}{ Pelvic tilt $\left(^{\circ}\right)$} \\
\hline maximum & $-0.331^{*}$ & $-0.611 *$ & $0.326^{*}$ & $-0.610^{*}$ \\
\hline minimum & $-0.446^{*}$ & $-0.474^{*}$ & 0.109 & $-0.547^{*}$ \\
\hline range & $0.286^{*}$ & -0.054 & 0.268 & 0.069 \\
\hline mean & $-0.457^{*}$ & $-0.560^{*}$ & 0.182 & $-0.635^{*}$ \\
\hline $\begin{array}{l}\text { Max hip extension in } \\
\text { stance }\left(^{\circ}\right)\end{array}$ & $-0.933^{*}$ & $-0.299 *$ & $-0.427^{*}$ & $-0.747^{*}$ \\
\hline $\begin{array}{l}\text { Max hip flexion in late } \\
\text { swing }\left(^{\circ}\right)\end{array}$ & -0.137 & $-0.740^{*}$ & $0.596^{*}$ & $-0.585^{*}$ \\
\hline \multicolumn{5}{|l|}{ Hip rotation $\left({ }^{\circ}\right)$} \\
\hline maximum & $-0.367^{*}$ & $-0.445^{*}$ & 0.142 & $-0.495^{*}$ \\
\hline minimum & $-0.388^{*}$ & $-0.423^{*}$ & 0.105 & $-0.442^{*}$ \\
\hline range & 0.098 & -0.003 & 0.077 & -0.072 \\
\hline mean & $-0.369^{*}$ & $-0.421^{*}$ & 0.117 & $-0.450^{*}$ \\
\hline TOC (\%) & $-0.324^{*}$ & -0.183 & -0.090 & $-0.417^{*}$ \\
\hline H3 (W/kg) & 0.135 & 0.004 & 0.106 & 0.009 \\
\hline HFI & $-0.467^{*}$ & $-0.503^{*}$ & 0.120 & $-0.646^{*}$ \\
\hline Cadence (No./min) & -0.133 & -0.001 & -0.100 & -0.109 \\
\hline Step length (cm) & 0.101 & -0.074 & 0.147 & -0.122 \\
\hline Walking speed $(\mathrm{cm} / \mathrm{s})$ & 0.040 & -0.022 & 0.051 & -0.122 \\
\hline
\end{tabular}

TOC, time of cross over in hip flexion/extension moment; $\mathrm{H} 3$, late swing power burst in hip joint flexion/extension power; HFI, hip flexor index; ${ }^{*}, \mathrm{p}<0.05$. burst of the hip flexor in late stance $(\mathrm{p}=0.627)$ (Table 5).

\section{Discussion}

The patients with cerebral palsy showed a shorter psoas length and smaller maximum hip extension in stance than the normal control group. The maximum psoas length was found to reflect the kinetic and kinematic data of hip motion. The hip flexor index showed satisfactory discriminant and convergent validity, showing a significant correlation with the psoas length. The result of the cross correlation revealed an excellent correlation between the maximum psoas length and maximum hip extension in the patients group (Table 6).

The patients with cerebral palsy showed a shorter maximum psoas length, larger pelvic tilt, and more sagittal pelvic motion than the normal control group. The maximum hip extension in stance was limited in the patient group, which was possibly caused by a

Table 5 Responsiveness of psoas length and gait parameters in patients with spastic diplegia

\begin{tabular}{llll}
\hline & Pre-operative & Post-operative & $\boldsymbol{p}$ \\
\hline $\begin{array}{l}\text { Pelvic tilt }\left({ }^{\circ}\right) \\
\quad\end{array}$ & & & \\
$\quad$ maximum & $21.9(4.9)$ & $18.8(4.9)$ & 0.008 \\
$\quad$ minimum & $12.6(5.9)$ & $12.9(5.0)$ & 0.897 \\
$\quad$ range & $9.4(3.5)$ & $5.9(2.1)$ & $<0.001$ \\
$\quad$ mean & $17.5(5.1)$ & $15.9(4.9)$ & 0.126 \\
$\begin{array}{l}\text { Max hip extension in } \\
\text { stance }\left({ }^{\circ}\right)\end{array}$ & $-0.5(6.1)$ & $4.6(7.5)$ & 0.001 \\
Max hip flexion in late & $50.4(5.9)$ & $42.9(5.1)$ & $<0.001$
\end{tabular}
swing $\left({ }^{\circ}\right)$

Hip rotation $\left({ }^{\circ}\right)$

$\begin{array}{llll}\text { maximum } & 12.8(8.2) & 10.0(4.9) & 0.107 \\ \text { minimum } & 0.2(9.2) & -5.2(7.0) & 0.016 \\ \text { range } & 12.6(4.1) & 15.3(4.6) & 0.015 \\ \text { mean } & 6.3(8.9) & 2.3(5.8) & 0.051\end{array}$

Psoas length (\%)

maximum

minimum

$99.2(1.3)$

$100.3(1.2)$

$<0.001$

range

$87.5(1.4)$

$89.3(1.6)$

$<0.001$

mean

$11.7(1.5)$

$11.1(1.9)$

0.158

TOC (\%)

H3 (W/kg)

$\mathrm{HFI}$

Cadence (No./min) $\quad 101.2(14.2) \quad 103.0(16.6) \quad 0.25$

$93.6(1.3)$

$95.0(1.2)$

$<0.001$

$28.2(11.5) \quad 24.6(12.3) \quad 0.173$

$\begin{array}{lll}0.3(0.4) & 0.5(2.0) & 0.627\end{array}$

$5.9(1.4) \quad 3.8(2.0) \quad<0.001$

Step length $(\mathrm{cm}) \quad 35.3(6.2) \quad 41.1(6.2) \quad<0.001$

Walking speed (cm/s) $59.9(13.5) \quad 71.1(15.8) \quad<0.001$

TOC, time of cross over in hip flexion/extension moment; $\mathrm{H} 3$, late swing power burst in hip joint flexion/extension power; HFI, hip flexor index. Data are presented as mean (SD).

All patients underwent bilateral femoral derotation osteotomy, rectus femoris transfer, distal hamstring lengthening, and tendo-Achilles lengthening as single event multilevel surgery. 
Table 6 Validity of the parameters for hip flexor contracture

\begin{tabular}{lllll}
\hline & $\begin{array}{l}\text { Discriminant validity } \\
\text { (effect-size r) }\end{array}$ & $\begin{array}{l}\text { Convergent validity in normal } \\
\text { control (correlation r) }\end{array}$ & $\begin{array}{l}\text { Convergent validity in CP } \\
\text { (correlation r) }\end{array}$ & $\begin{array}{l}\text { Responsiveness } \\
\text { (effect-size r) }\end{array}$ \\
\hline $\begin{array}{l}\text { Maximum pelvic tilt } \\
\left(^{\circ}\right)\end{array}$ & $* * * *$ & $* *$ & $* *$ \\
$\begin{array}{l}\text { Max hip extension in } \\
\text { stance }\left({ }^{\circ}\right)\end{array}$ & $* * * *$ & $* * *$ & $* * * *$ \\
$\mathrm{HFl}$ & & $* * * *$ & $* * *$ & $* *$ \\
$\begin{array}{l}\text { Maximum psoas } \\
\text { length (\%) }\end{array}$ & $* * * *$ & + & + & $* * *$ \\
\hline
\end{tabular}

$\mathrm{HFI}$, hip flexor index.

*, 0-0.2; ${ }^{* *}, 0.2-0.4$; $^{* *}, 0.4-0.6$; ${ }^{* * *}, 0.6-0.8$; $^{* * * *}, 0.8-1.0$.

$t$, convergent validity was the correlation coefficient with maximum psoas length.

shorter psoas length. However, the range of psoas lengths was similar in the patients and control group suggesting that muscle excursion was not significantly different. The kinetic variable, including the time of crossover in the hip flexion-extension moment and the power burst of the hip flexor in late stance, were similar in the patients and controls. In this study, the maximum psoas length, hip flexor index and sagittal pelvic motion showed favorable discriminant validity. The correlation coefficient between the maximum psoas length and maximum hip extension in stance was $-0.420(\mathrm{p}=0.065)$ in the control group whereas it was -0.933 in the patients $(\mathrm{p}<0.001)$. The shortened maximum psoas length in the patients appeared to limit the maximum extension of the hip joint. However, the maximum psoas length might not have been the limiting factor in maximum hip extension in the control group. This could have cause different correlation coefficients of the two groups between maximum psoas length and maximum hip extension in stance.

It has been reported that the psoas length could be confounded by the femoral anteversion [30], which is supported by the results of this study. The psoas length increased post-operatively, even though no psoas procedures had been performed in addition to femoral derotation osteotomy. Therefore, femoral derotation osteotomy may improve the dynamic psoas length possibly by moving the lesser trochanter forward. However, this requires further examination.

This study had some limitations. First, although small changes in the kinetic and kinematic variables in the study were statistically significant, they may have been due to marker placement variability, despite this being performed by a single experienced operator. Second, the $3 \mathrm{D}$ psoas length did not reflect the lumbar spinal motion which could affect the real psoas length significantly because the model did not contain the trunk marker sets. Therefore, the 3D modeled psoas length might not be as accurate as expected.

\section{Conclusions}

The patients with cerebral palsy showed a shorter psoas length than the normal control group. The hip flexor index and psoas length showed good discriminant validity. There was an excellent correlation between the maximum psoas length and maximum hip extension in the patients group. There was evidence that estimated psoas length could be improved after femoral derotation osteotomy, even though no psoas procedure had been performed.

\section{Acknowledgements}

The authors wish to thank Seon Boo, BS and Myoung YI Park, BS for the technical support and advice, and Mi Sun Ryu for collecting the data. This study was conducted at Seoul National University Bundang Hospital. There was internal funding for this study from Seoul National University Bundang Hospital (SNUBH research fund 02-2008-030).

\section{Author details}

'Department of Orthopedic Surgery, Synergy Hospital, 115-17 NonhyunDong, Kangnam-Gu, Seoul, 135-010, Republic of Korea. ${ }^{2}$ Department of Orthopedic Surgery, Seoul National University Bundang Hospital, 300 GumiDong, Bundang-Gu,Sungnam, Kyungki 463-707, Republic of Korea. ${ }^{3}$ Department of Orthopedic Surgery, Dongguk University Ilsan Hospital, 814 Siksa-Dong, Ilsandong-Gu, Koyang, Kyungki 410-773, Republic of Korea.

\section{Authors' contributions}

CYC, MSP, and KML have made substantial contributions to conception and design. SJC, DGK, and SHL have been involved in acquisition of data, analysis and interpretation of data. SJC, KML and MSP drafted the manuscript. All authors read and approved the manuscript.

\section{Competing interests}

The authors declare that they have no competing interests.

Received: 20 May 2010 Accepted: 23 January 2011

Published: 23 January 2011

\section{References}

1. Bleck EE: Postural and gait abnormalities caused by hip-flexion deformity in spastic cerebral palsy. Treatment by iliopsoas recession. J Bone Joint Surg Am 1971, 53:1468-1488.

2. Dostal WF, Andrews JG: A three-dimensional biomechanical model of hip musculature. J Biomech 1981, 14:803-812.

3. Feldkamp M, Denker P: Importance of the iliopsoas muscle in soft-tissue surgery of hip deformities in cerebral palsy children. Arch Orthop Trauma Surg 1989, 108:225-230.

4. Bialik GM, Pierce R, Dorociak R, Lee TS, Aiona MD, Sussman MD: Iliopsoas tenotomy at the lesser trochanter versus at the pelvic brim in 
ambulatory children with cerebral palsy. J Pediatr Orthop 2009, 29:251-255.

5. Matsuo T, Hara H, Tada S: Selective lengthening of the psoas and rectus femoris and preservation of the iliacus for flexion deformity of the hip in cerebral palsy patients. J Pediatr Orthop 1987, 7:690-698.

6. Harada T, Ebara S, Anwar MM, Kajiura I, Oshita S, Hiroshima K, Ono K: The lumbar spine in spastic diplegia. A radiographic study. J Bone Joint Surg Br 1993, 75:534-537.

7. Hoffer MM: Management of the hip in cerebral palsy. J Bone Joint Surg Am 1986, 68:629-631.

8. Houkom JA, Roach JW, Wenger DR, Speck G, Herring JA, Norris EN: Treatment of acquired hip subluxation in cerebral palsy. J Pediatr Orthop 1986, 6:285-290.

9. Zwick EB, Saraph V, Zwick G, Steinwender C, Linhart WE, Steinwender G Medial hamstring lengthening in the presence of hip flexor tightness in spastic diplegia. Gait Posture 2002, 16:288-296.

10. Sutherland DH, Zilberfarb JL, Kaufman KR, Wyatt MP, Chambers HG: Psoas release at the pelvic brim in ambulatory patients with cerebral palsy: operative technique and functional outcome. J Pediatr Orthop 1997, 17:563-570.

11. Morais Filho MC, de Godoy W, Santos CA: Effects of intramuscular psoas lengthening on pelvic and hip motion in patients with spastic diparetic cerebral palsy. J Pediatr Orthop 2006, 26:260-264.

12. Delp SL, Arnold AS, Piazza SJ: Graphics-based modeling and analysis of gait abnormalities. Biomed Mater Eng 1998, 8:227-240.

13. Schutte LM, Hayden SW, Gage JR: Lengths of hamstrings and psoas muscles during crouch gait: effects of femoral anteversion. J Orthop Res 1997, 15:615-621.

14. Delp SL, Arnold AS, Speers RA, Moore CA: Hamstrings and Psoas lengths during normal and crouch gait: implications for muscle tendon surgery. J Orthop Res 1996, 14:144-151.

15. Van der Krogt MM, Doorenbosch CA, Harlaar J: Muscle length and lengthening velocity in voluntary crouch gait. Gait Posture 2007, 26:532-538.

16. Palisano R, Rosenbaum P, Walter S, Russell D, Wood E, Galuppi B: Development and reliability of a system to classify gross motor function in children with cerebral palsy. Dev Med Child Neurol 1997, 39:214-223.

17. Rodda JM, Graham HK, Carson L, Galea MP, Wolfe R: Sagittal gait patterns in spastic diplegia. J Bone Joint Surg Br 2004, 86:251-258.

18. Thomas HO: Diseases of hip, knee, and ankle joints, with their deformities, treated by new and efficient method. Liverpool: Dobb; 1876.

19. Schwartz MH, Novacheck TF, Trost J: A tool for quantifying hip flexor function during gait. Gait Posture 2000, 12:122-127.

20. Delp SL, Loan JP, Hoy MG, Zajac FE, Topp EL, Rosen JM: An interactive graphics-based model of the lower extremity to study orthopaedic surgical procedures. IEEE Trans Biomed Eng 1990, 37:757-767.

21. Campbell DT, Fiske DW: Convergent and discriminant validation by the multitrait-multimethod matrix. Psychol Bull 1959, 56:81-105.

22. Jewell DV: Guide to Evidence-based physical therapy practice. Sudbury: Jones \& Bartlett Learning;, 22010.

23. Cohen J: Statistical power analysis for the behavioral sciences. Hillsdale, NJ, Lawrence Earlbaum Associates; 21988.

24. Stouthard MEA, Hoogstraten J, Mellenbergh GJ: A study on the convergent and discriminant validity of the dental anxiety inventory. Behav Res Ther 1995, 33:589-595.

25. Quinnell FA, Hynan MT: Convergent and discriminant validity of the perinatal PTSD questionnaire (PPQ): A preliminary study. J Traumatic Stress 1999, 12:193-199.

26. Sagi A, Van IJzendoorn MH, Scharf M, Koren-Karie N, Joels T, Mayseless O: Stability and Discriminant validity of the adult attachment interview: A psychometric study in young Israeli adults. Dev Psychol 1994, 30:771-777.

27. Westen D, Rosenthal R: Quantifying construct validity: Two simple measures. J Pers Soc Psychol 2003, 84:608-618.

28. Ware JEJ, Snow KK, Kosinski M, Gandek B: SF-36 Health Survey Manual and Interpretation Guide. Boston; USA, New England Medical Center; 1993.

29. Staquet MJ, Hays RD, Fayers PM: Quality of life assessment in clinical trials. New York, Oxford Unversity Press; 1998.

30. Schutte LM, Hayden SW, Gage JR: Lengths of hamstrings and psoas muscles during crouch gait: effects of femoral anteversion. J Orthop Res 1997, 15:615-621. doi:10.1186/1743-0003-8-4

Cite this article as: Choi et al:: Validity of gait parameters for hip flexor contracture in patients with cerebral palsy. Journal of NeuroEngineering and Rehabilitation 2011 8:4

\section{Submit your next manuscript to BioMed Central and take full advantage of:}

- Convenient online submission

- Thorough peer review

- No space constraints or color figure charges

- Immediate publication on acceptance

- Inclusion in PubMed, CAS, Scopus and Google Scholar

- Research which is freely available for redistribution 